OPEN ACCESS

Edited by:

Hubing Shi,

Sichuan University, China

Reviewed by:

Jinhua Wang,

Chinese Academy of Medical Sciences and Peking Union Medical

College, China

Yeye Guo,

Central South University, China

${ }^{*}$ Correspondence:

Lang Xie

langxiezj@hotmail.com

tThese authors have contributed equally to this work

Specialty section:

This article was submitted to Pharmacology of Anti-Cancer Drugs,

a section of the journal

Frontiers in Pharmacology

Received: 15 September 2018 Accepted: 06 February 2019

Published: 28 February 2019

Citation:

Li Y, He M, Zhou Y, Yang C, Wei S,

Bian X, Christopher O and Xie L (2019) The Prognostic and Clinicopathological Roles of PD-L1

Expression in Colorectal Cancer: A Systematic Review and Meta-Analysis.

Front. Pharmacol. 10:139.

doi: 10.3389/fphar.2019.00139

\section{The Prognostic and}

\section{Clinicopathological Roles of PD-L1 Expression in Colorectal Cancer: A Systematic Review and Meta-Analysis}

\author{
Yan $\mathrm{Li}^{1,2 \dagger}$, Meizhi He ${ }^{2 \dagger}$, Yaoyao Zhou ${ }^{2}$, Chen Yang ${ }^{3}$, Shuyi Wei ${ }^{2}$, Xiaohui Bian ${ }^{2}$, \\ Odong Christopher ${ }^{3}$ and Lang Xie ${ }^{\text {* }}$
}

${ }^{1}$ Department of General Surgery, Zhujiang Hospital of Southern Medical University, Guangzhou, China, ${ }^{2}$ The Second School of Clinical Medicine, Southern Medical University, Guangzhou, China, ${ }^{3}$ The First School of Clinical Medicine, Southern Medical University, Guangzhou, China

Background: Studies evaluating the prognostic significance of programmed death-ligand 1 (PD-L1) expression in colorectal cancer (CRC) are limited and remain controversial. This meta-analysis was conducted in order to evaluate the clinicopathological and prognostic significance of PD-L1 expression in CRC patients.

Methods: A comprehensive search was performed against the Medline/PubMed, Embase, Cochrane Library, Web of Science (WoS) and Scopus databases. Data were extracted with name of the first author, year of publication, country of origin, tumor type, number of cases, staining method, cut-off values, PD-L1 positive expression, clinicopathological parameters, outcome, and quality assessment score, and statistical analysis was conducted using Review Manager Version 5.3 (Revman the Cochrane Collaboration; Oxford, England) and STATA version 14 (Stata Corporation; College Station, TX, USA).

Results: Ten studies were included in this meta-analysis, in which the pooled hazard ratio $(H R)$ showed that PD-L1 expression in tumor cells was significantly associated with a poor overall survival $(H R=1.50,95 \% \mathrm{Cl} 1.05-2.13, P=0.03)$. The pooled HR for disease-free survival (DFS) indicated that PD-L1 expression was significantly associated with shorter DFS ( $\mathrm{HR}=2.57,95 \% \mathrm{Cl} 1.40-4.75, P=0.002)$. The pooled odds ratios (ORs) showed that $\mathrm{PD}-\mathrm{L} 1$ expression was associated with poor differentiation $(\mathrm{OR}=3.47,95 \% \mathrm{Cl} 1.37-8.77, P=0.008)$ and right colon cancer $(\mathrm{OR}=2.38,95 \% \mathrm{Cl}$ 1.57-3.60, $P<0.0001)$. However, the expression of PD-L1 was independent of gender, age, tumor size, tumor stage, lymph node metastasis, and tumor-node metastasis stage.

Conclusion: This meta-analysis indicated that a high level of PD-L1 expression might be a biomarker for a poor prognosis in CRC patients. This information may be helpful for clinicians to stratify CRC patients for anti-PD-1/PD-L1 therapy, particularly patients with microsatellite instability high (MSI-H).

Keywords: colorectal cancer, PD-L1/ PD-1, prognostic, clinicopathological, meta-analysis 


\section{INTRODUCTION}

Globally, colorectal cancer (CRC) is the third leading cause of cancer (Siegel et al., 2017). Although cancer screening programs and the standardization of preoperative and postoperative care have reduced mortality associated with a CRC diagnosis (Welch and Robertson, 2016), CRC is still a leading cause of cancerrelated deaths worldwide, for it has a poor prognosis in its malignant stages and recurrence is common. Therefore, it is essential to identify new biomarkers to improve clinical decisionmaking and patient outcomes.

As one of the most possible newly biomarkers to evaluate cancer patients' outcomes, programmed death 1 (PD-1) is an immune-inhibitory receptor that is expressed on the surface of activated $\mathrm{T}$ cells as a result of persistent inflammatory stimuli (Inaguma et al., 2016; Zou et al., 2016). PD-L1 is expressed by T and $\mathrm{B}$ cells, macrophages and dendritic cells and its expression implies a weakened host immune response and consequent a poor prognosis (Hansen et al., 2009). The binding of PD-L1 to PD-1 can attenuate the cellular immune response by reducing $\mathrm{T}$ cells apoptosis or exhaustion. Blockade of the PD-1/PDL1 pathway with monoclonal antibodies is a highly promising therapy and prominent clinical benefits of this checkpointblockade were observed in recent clinical trials (Zheng and Zhou, 2015; Wang et al., 2018).

Positive PD-L1 expression has been associated with significantly poor prognoses; however, studies evaluating the prognostic significance of PD-L1 expression in CRC are limited and remain controversial. Therefore, we conducted a comprehensive meta-analysis to evaluate the clinicopathological and prognostic significance of PD-L1 expression in CRC patients.

\section{MATERIALS AND METHODS}

\section{Literature Search}

Two authors (M. Z. He and Y. Y. Zhou) independently conducted comprehensive literature searches of published articles using the Medline/PubMed, Embase, Cochrane Library, WoS and Scopus databases. The endpoint for search items was July 21, 2018. The following keywords were used: ("colorectal" OR "colorectum" OR "colon" OR "Rectum" OR "Rectal” OR "large intestine") AND ("adenocarcinoma?" OR "tumor?" OR “neoplasm?” OR “carcinoma?” OR “cancer?” OR “malignant”) AND ("Programmed Cell Death 1 Receptor" OR "CD279 Antigen" OR "PD-1" OR "B7-H1 Antigen” OR "Programmed Cell Death 1 Ligand 1" OR “PD-L1 “OR “CD 274”). Titles and abstracts were screened through NoteExpress and any discrepancies were resolved by mutual discussion.

Abbreviations: PD-L1, programmed death-ligand 1; CRC, colorectal cancer; WoS, wet of science; HR, hazard ratio; TCs, tumor cells; OS, overall survival; DFS, disease-free survival; ORs, odds ratios; T stage, tumor stage; TNM, tumornode-metastasis; MSI-H, microsatellite instability high; PD-1, programmed death 1; IHC, immunohistochemistry; CIs, confidence intervals; NOS, newcastleottawa quality assessment; IRS, immunoreactivity score; TILs, tumor-infiltrating lymphocytes; CTLs, CD8+ cytotoxic T lymphocytes; CTLA4, CTL-associated antigen 4; IDO1, indoleamine 2,3-dioxygenase 1; TIME, tumor immunity in the microEnvironment; $\mathrm{mPD}-\mathrm{L} 1, \mathrm{PD}-\mathrm{L} 1$ promoter methylation.

\section{Eligibility Criteria}

The criteria for inclusion were: (1) All patients were histologically confirmed as having CRC and had not received adjuvant chemotherapy before surgery; (2) PD-L1 expression was detected by immunohistochemistry (IHC); (3) Studies showed a correlation between PD-L1 expression with clinicopathological features and/ or prognoses; (4) Articles were published as a full paper in English. The criteria for exclusion were: (1) Case reports, reviews and letters; (2) The main content did not evaluate the relationship of PDL1 expression with clinicopathological features and/ or prognoses; (3) duplications and studies without eligible data. When duplicate publications were identified, only the article with the newest and most comprehensive information was included.

\section{Data Extraction and Quality Assessment}

The following information from the included articles was extracted by two reviewers (M. Z. He and Y. Y. Zhou): name of the first author, year of publication, country of origin, tumor type, number of cases, staining method, cutoff values, PD-L1 positive expression, clinicopathological parameters, outcome, and quality assessment score. Any disagreements between the two reviewers were resolved by consensus involving a third reviewer (Y. Li). Outcome parameters comprised OS, DFS and recurrence-free survival (RFS). The HRs and 95\% confidence intervals (CIs) were evaluated for outcome parameters. If the HRs were not available, we extracted data from survival curves or contacted the corresponding authors.

According to the Newcastle-Ottawa Quality Assessment (NOS), a quality assessment was independently carried out for the included articles by two authors (M.Z. He and Y. Y. Zhou). Discrepancies in scoring were resolved by discussion and consensus. The NOS consists of the following three parameters of quality: selection, comparability and outcome. The maximum NOS score is nine points, with studies scoring greater than six considered to be of high quality (Stang, 2010).

\section{Statistical Methods}

Pooled HRs and 95\% CIs were calculated to evaluate the association between PD-L1 positive expression with OS, DFS, RFS and clinicopathological parameters. Heterogeneity among studies was evaluated using the Chi-squared test and $I^{2}$. A random-effects model was used when there was evidence of significant heterogeneity $\left(I^{2}>50 \%\right.$ or $P$-value $\left.<0.1\right)$. In all other cases, a fixed-effects model was used. Potential publication bias was assessed through Egger's and Begg's tests. The statistical analysis was conducted using Review Manager Version 5.3 (Revman the Cochrane Collaboration; Oxford, England) and STATA version 14 (Stata Corporation; College Station, TX, USA). All $P$-values and 95\% CIs were two-sided, and $P$-values $<0.05$ were considered to be statistically significant. 


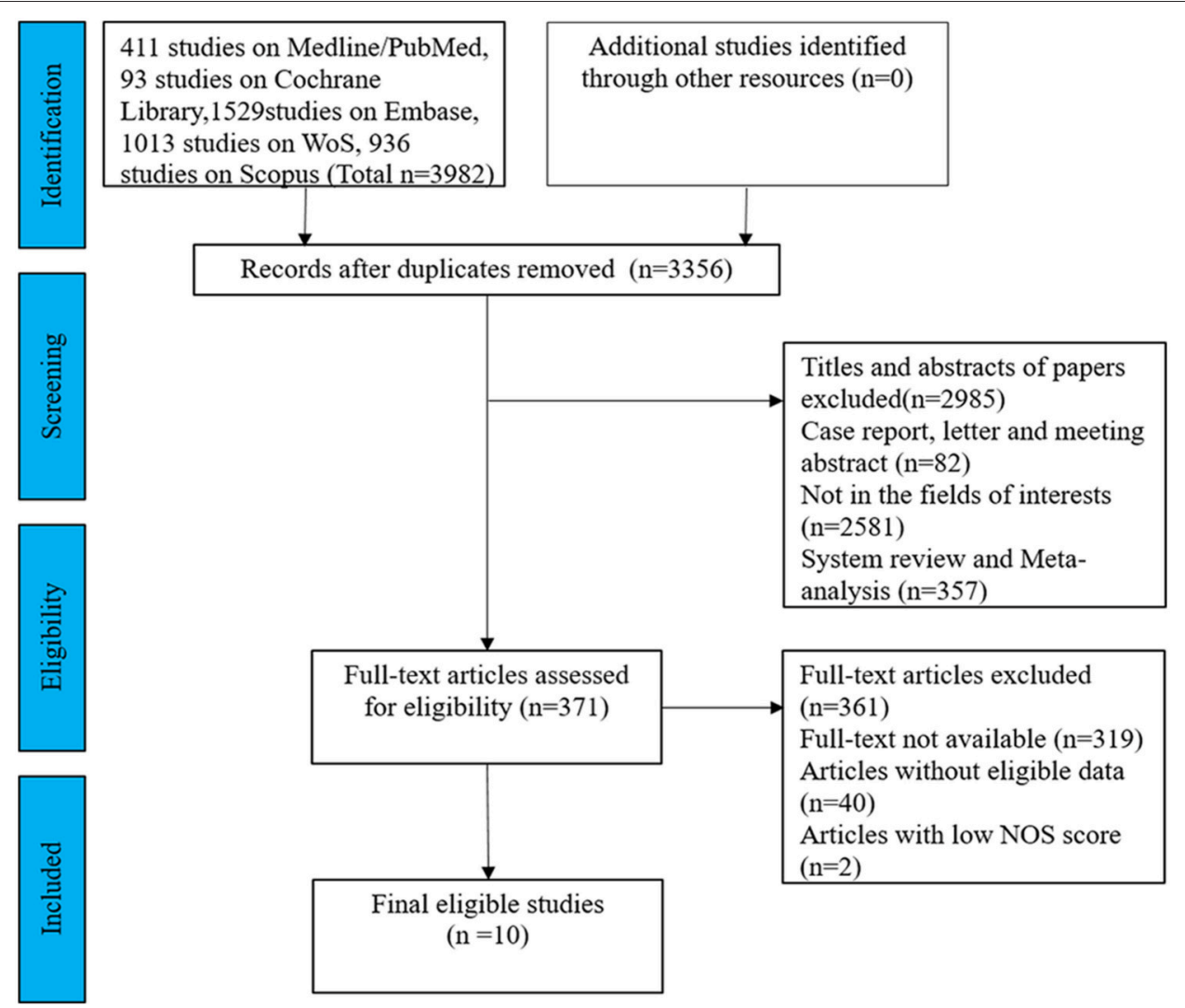

FIGURE 1 | Flow diagram of the study selection process in this meta-analysis.

\section{RESULTS}

\section{Search Results and Study Characteristics}

After exclusion of 626 duplicates, 3,356 articles about PD-1/PDL1 in colorectal cancer were identified from a primary system literature search in the Medline/PubMed, Embase, Cochrane Library, WoS, and Scopus databases. The titles and abstracts of the remaining articles were screened, and 2,985 records were rejected because they were case reports, letters, meeting, reviews or not in the fields of interests. We read 371 records for further assessment. Among them, 319 full-text articles were not available, another 40 lacked eligible data, and two scored lower than 6 on the NOS. Finally, 10 articles were included in this meta-analysis. A flowchart of the literature selection is shown in Figure 1.

The characteristics of the 10 included studies are listed in Table 1. These included studies were generally of high quality, with NOS scores ranging from six to eight. All 10 studies were retrospective and published between 2013 and 2018. In total, 10 studies comprising 2,131 patients were included in the pooled analysis and all selected studies used IHC assays to evaluate PD-L1 expression in tumor cells and /or TILs. Each article had an independent cut-off value used to define the criterion for PD-L1 positive. Six studies provided OS data (Shi et al., 2013; Zhu et al., 2015; Li et al., 2016; Enkhbat et al., 2018; Lee S. J. et al., 2018; Liu et al., 2018), three studies included DFS data (Enkhbat et al., 2018; Lee K. S. et al., 2018; Lee S. J. et al., 2018) and three studies included RFS data (Lee et al., 2016; Wang et al., 2016, 2017). In addition, HRs and $95 \%$ CIs were abstracted directly from the 10 included studies.

\section{Association Between PD-L1 Expression and Prognostic Parameters}

We evaluated the association between PD-L1 expression and prognostic parameters (OS, DFS and RFS). The pooled HR for OS in TC from six studies, involving 1,131 patients, showed that PD-L1 expression was significantly associated with poor OS in CRC $(\mathrm{HR}=1.50,95 \% \mathrm{CI} 1.05-2.13, P=0.03$; see Figure 2A). When we took Immunoreactivity score (IRS) $\geq 4$ as the cut-off value, we found shorter survival in the PD-L1 positive group $(\mathrm{HR}=2.65,95 \% \mathrm{CI} 1.44-4.86, P=$ 0.002; see Figure 2B). The pooled HR for DFS in TC with 452 patients indicated that PD-L1 expression was significantly associated with shorter DFS ( $\mathrm{HR}=2.57,95 \% \mathrm{CI} 1.40-4.75$, $P=0.002$; see Figure 2C). The pooled HR for RFS in TC with 657 patients $(\mathrm{HR}=2.38,95 \% \mathrm{CI} 1.14-4.96, P=0.02$; see Figure 2D) as well as the pooled HR for RFS in tumor-infiltrating lymphocytes (TILs) with $516 \mathrm{CRC}$ patients $(\mathrm{HR}=1.79,95 \% \mathrm{CI}$ 
TABLE 1 | Main characteristics of the studies included for meta-analysis.

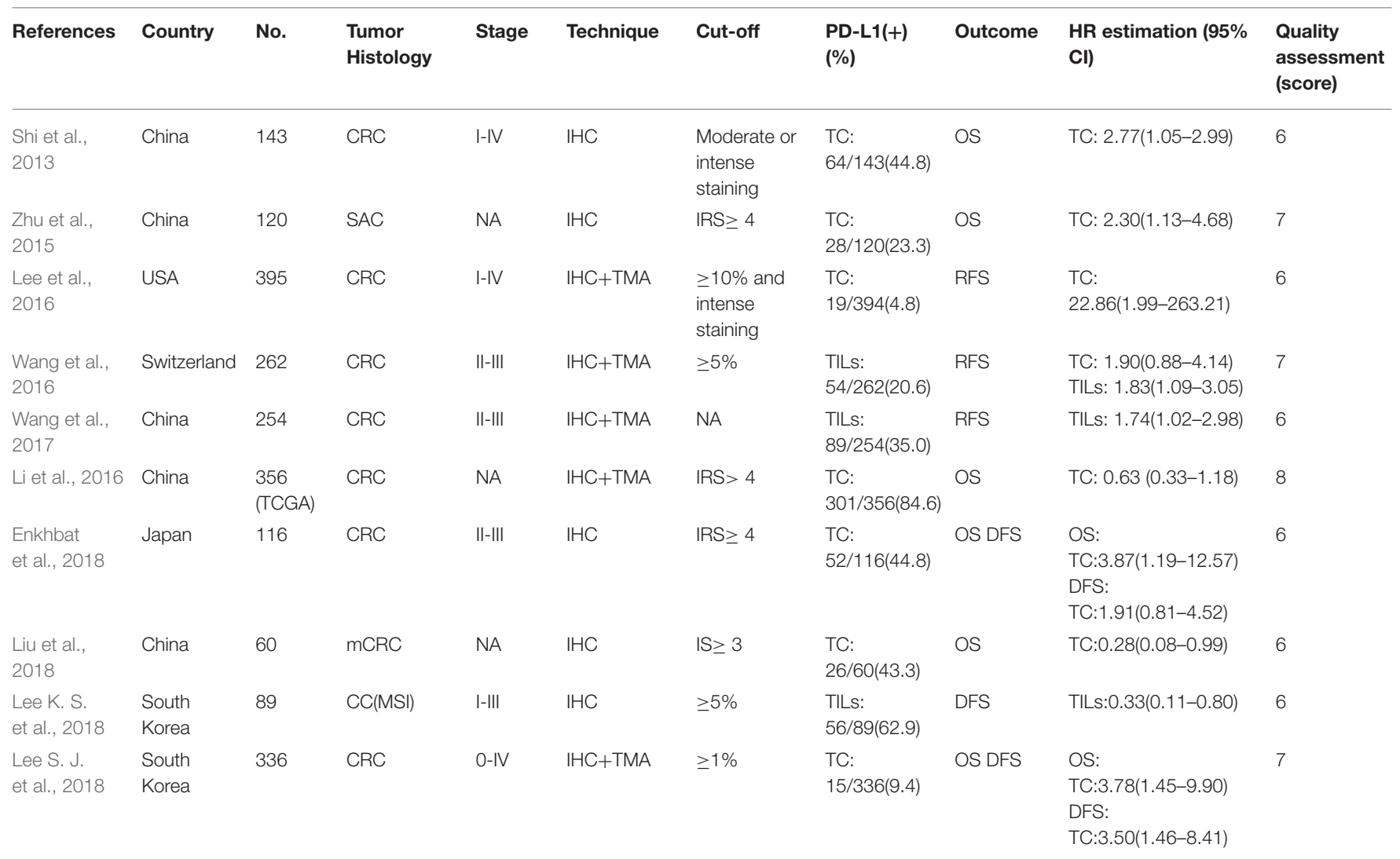

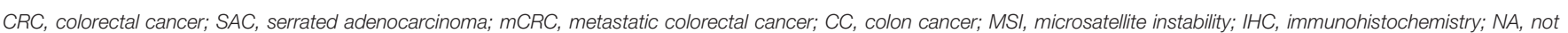

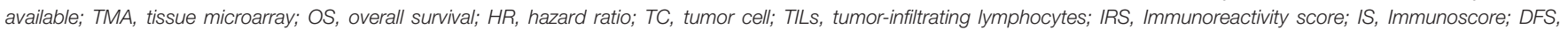
disease-free survival; RFS, recurrence-free survival.

1.23-2.95, $P=0.002$; see Figure $2 \mathrm{E}$ ) showed that PD-L1 expression was significantly associated with poor RFS both in TC and TILs.

\section{Association Between PD-L1 Expression and Clinicopathological Characteristics Gender}

The association between PD-L1 expression and gender was evaluated in eight studies, comprising 3,477 patients. $320(31.37 \%)$ of 1,020 male patients and $241(31.42 \% \%)$ of 767 female patients were PD-L1 expression positive. The pooled OR showed that there was no significant association found between $\mathrm{PD}-\mathrm{L} 1$ expression and gender $(\mathrm{OR}=1.00,95 \% \mathrm{CI} 0.76-1.31, P=$ 0.98; see Figure 3A).

\section{Age}

We evaluated the association between PD-L1 expression and age in a total of 405 patients from two studies. 49 (26.78\%) of 183 younger patients ( $<60$ years of age) were PD-L1 expression positive and $69(31.08 \%)$ of 222 older patients ( $\geq 60$ years of age) were PD-L1 expression positive. There was no significant association found between PD-L1 expression and age $(\mathrm{OR}=1.41$, 95\% CI 0.90-2.23, $P=0.13$; see Figure 3B).

\section{Cancer Location}

The association between PD-L1 expression and cancer location was analyzed in six studies with a population of 1,025 patients. Of 344 right colon cancer patients, 65 (18.90\%) were PD-L1 expression positive, while $77(11.31 \%)$ in 681 left colon/rectum cancer patients. The pooled OR showed a significant association between PD-L1 expression and cancer location $(\mathrm{OR}=2.38,95 \%$ CI 1.57-3.60, $P<0.0001$; see Figure 3C).

\section{Differentiation}

Of 1,066 well/moderately differentiated tumors, 159 (14.92\%) were PD-L1 expression positive. Of 154 poorly differentiated tumors, 49 (34.82\%) were PD-L1 expression positive. The pooled OR showed that PD-L1 expression was significantly associated with differentiation based on pooled data from five studies (OR $=3.47$, 95\% CI 1.37-8.77, $P=0.008$; see Figure 3D).

\section{Tumor Size}

Only two studies, including 382 colorectal cancer patients, analyzed the subgroup of tumor size based on the cut-off value of $5 \mathrm{~cm} .36(25.17 \%)$ of 143 patients with large tumors $(\geq 5 \mathrm{~cm})$ and $48(20.01 \%)$ of 239 patients with small tumors $(<5 \mathrm{~cm})$ were PDL1 expression positive. The pooled results carried out in a fixed effect model, showed that there was no significant association 


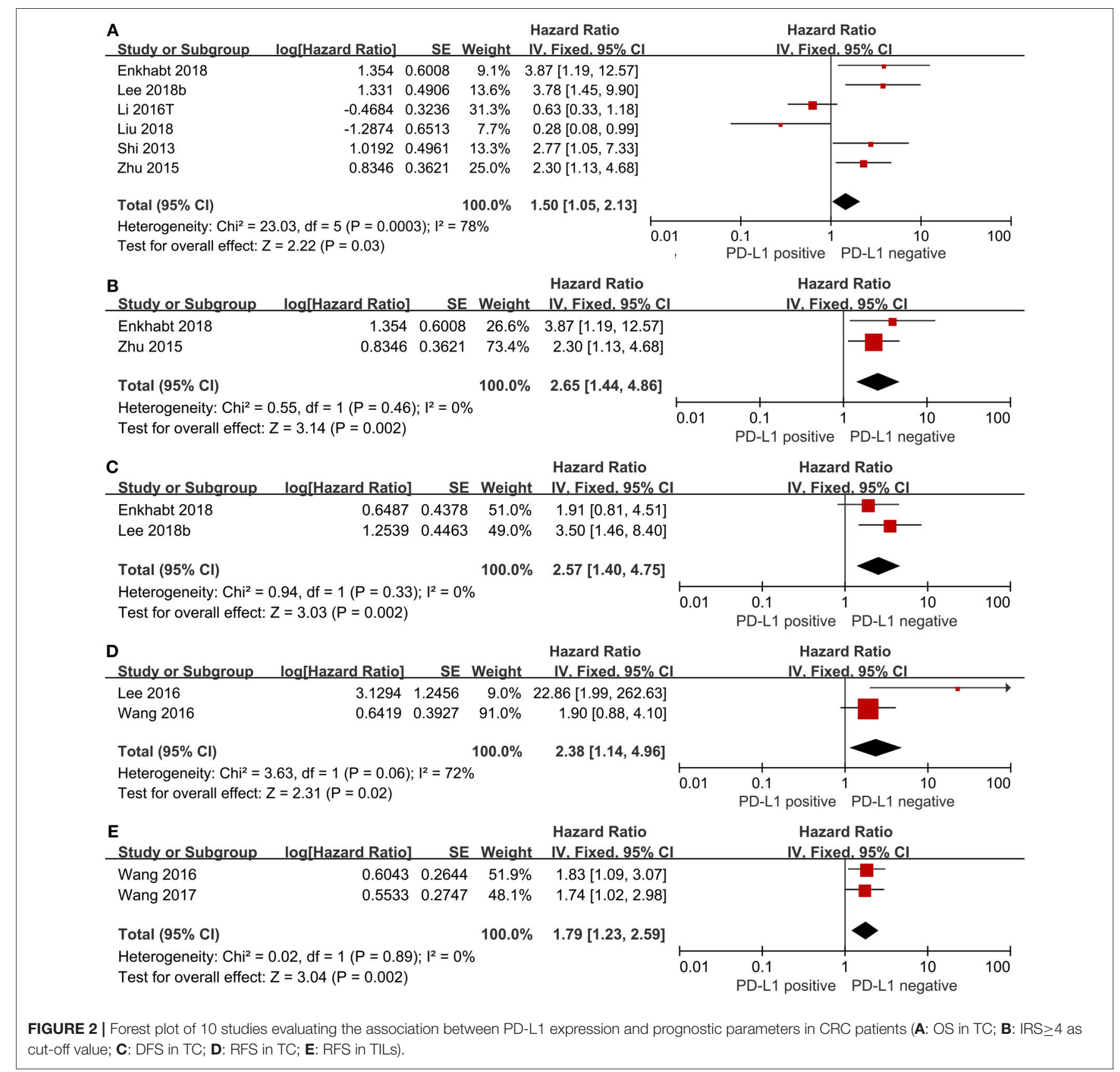

between PD-L1 expression and tumor size $(\mathrm{OR}=1.31,95 \% \mathrm{CI}$ $0.80-2.14, P=0.29$; see Figure 3E).

\section{T Stage}

We evaluated the association between PD-L1 expression and T stage in 1,716 patients. Of 283 Tis-T2 stage patients, 82 (28.98\%) were PD-L1 expression positive and 454 (31.68\%) of 1,433 T3-T4 stage patients were PD-L1 expression positive. The pooled HR showed that there was no significant association between PD-L1 expression and $\mathrm{T}$ stage $(\mathrm{OR}=1.02,95 \% \mathrm{CI} 0.68-1.54, P=0.93$; see Figure 3F).

\section{Lymph Node Metastasis}

The association between PD-L1 expression and lymph node metastasis was evaluated in six studies (1,589 patients). The pooled $\mathrm{OR}$ indicated that there was no significant association found between PD-L1 expression and lymph node metastasis $(\mathrm{OR}=1.23,95 \% \mathrm{CI} 0.71-2.12, P=0.46$; see Figure 3G).

\section{TNM Stage}

Six studies, involving 1,329 patients, evaluated the association between PD-L1 expression and TNM stage in a fixed effects model. 138 (21.26\%) of 649 stage I-II patients and $122(17.94 \%)$ of 680 stage III-IV patients were PD-L1 expression positive. The 


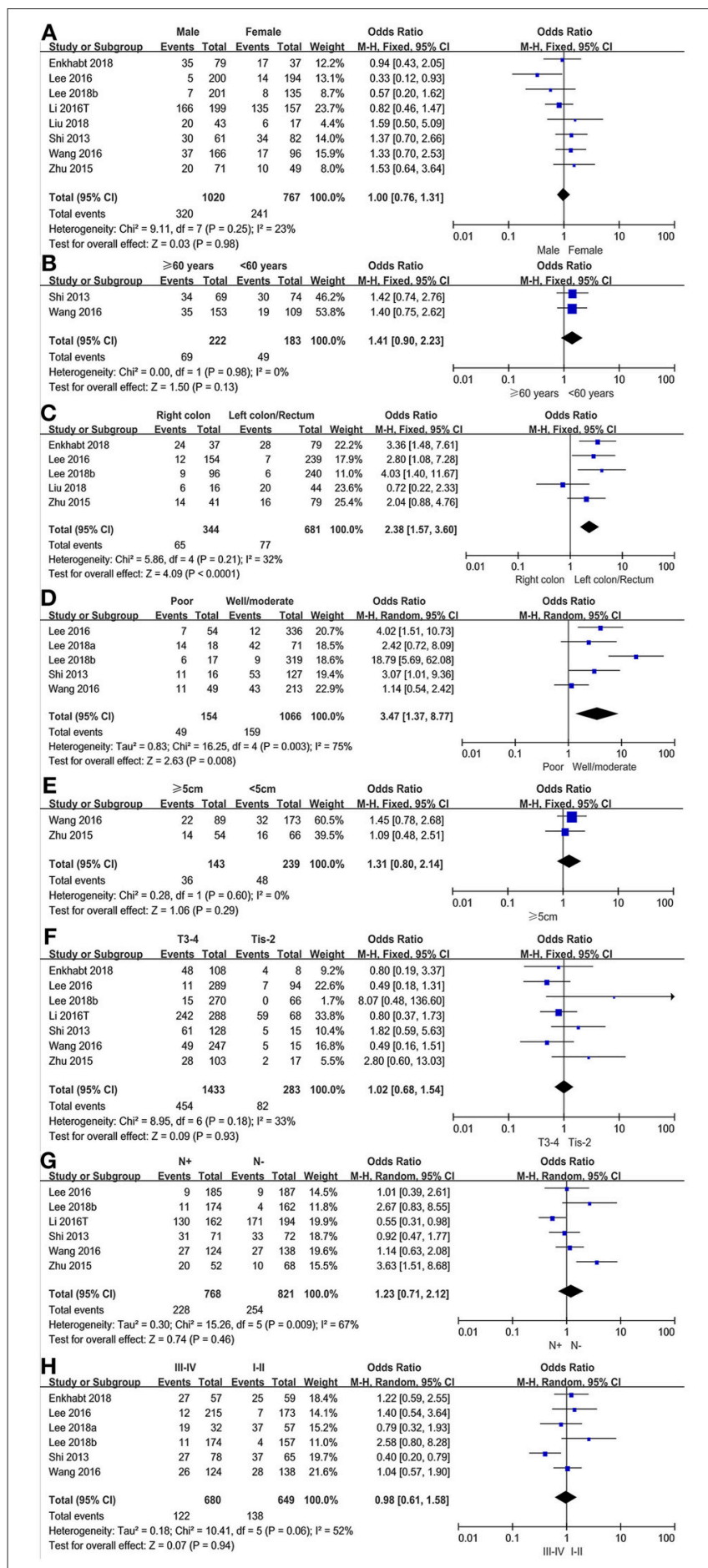

FIGURE 3 | Forest plots for the association of PD-L1 expression with clinicopathological features in $\mathrm{CRC}$ patients (A: gender; B: age; C: cancer location; D: differentiation; E: tumor size; F: T stage; G: lymph node metastasis; $\mathbf{H}$ : TNM stage).

pooled result showed no significant association found between PD-L1 expression and TNM stage $(\mathrm{OR}=0.98,95 \%$ CI $0.61-1.58$, $P=0.94$; see Figure $3 \mathbf{H}$ ).
Heterogeneity was identified in the analysis of PD-L1 expression with cancer location $\left(P=0.73, I^{2}=82 \%\right)$ and lymph node metastasis $\left(P=0.46, I^{2}=67 \%\right)$. Therefore, a random effects model was used in the above analyses and other subgroup analyses were performed in a fixed effects model.

\section{Publication Bias}

Egger's and Begg's tests showed that no publication bias influencing the HRs for OS was observed in the six studies (Figure 4). The $P$-values for these tests were 0.683 and 1.000 , respectively. In addition, the funnel plots showed no publication bias for gender or $\mathrm{T}$ stage (Figure 5).

\section{DISCUSSION}

In the present meta-analysis of the clinicopathological and prognostic significance PD-L1 expression in CRC, we found that PD-L1 expression was significantly associated with poor OS in TC. In addition, the pooled results of RFS and DFS showed that PD-L1 expression was significantly correlated with unfavorable clinical outcomes. Poor differentiation and right colon CRC tumors suggested a poor prognosis. The expression of PD-L1 was independent of gender, age, tumor size, $\mathrm{T}$ stage, lymph node metastasis, and TNM stage. To our knowledge, this comprehensive meta-analysis is the first to evaluate the association of PD-L1 expression with clinicopathological characteristics and prognostic parameters in colorectal cancer.

During the process of study of selection, the study of Droeser et al. (2013) was excluded for it included unselected, nonconsecutive, primary, sporadic colorectal cancers, and the data of the included articles in this meta-analysis were satisfied with a more rigorous standards, which excluded the patients receiving adjuvant chemotherapy before surgery, diagnosis of gastrointestinal stromal tumor or lymphoma, diagnosis with additional cancers. It is well-known that accurate results were based on the rigorous exclusion criteria in retrospective study. Among the OS data in six included studies, one study showed contradictory results showing that PD-L1 positive expression was significantly associated with better OS. This study was not the only one to report a positive prognostic impact of PDL1 expression. Sabatier et al. (Schalper et al., 2014) evaluated PD-L1 expression in 5,454 breast cancer cases and found that positive PD-L1 expression was associated with better metastasisfree survival and improved response to chemotherapy. However, the pooled result showed a significant correlation of PD-L1 expression and poor prognostic outcomes was supported by other articles reporting poorer outcomes in renal cell carcinoma, non-small cell lung cancer (Wang et al., 2015) and osteosarcoma (Lussier et al., 2015). This was because of the complex function of PD-L1 in the initiation and growth of CRC.

PD-L1 is upregulated by many inflammatory mediators and cytokines (Keir et al., 2006, 2008; Okazaki and Honjo, 2006) and PD1/PD-L1 binding can induce activated T cell apoptosis, exhaustion, and interleukin-10(IL-10) expression as a negative feedback system (Zou et al., 2016). Moreover, PD-L1 expression can help tumor cells to evade immunosurveillance and enhance the function of Tregs in CRC (Lu et al., 2011; Toh et al., 2016). 


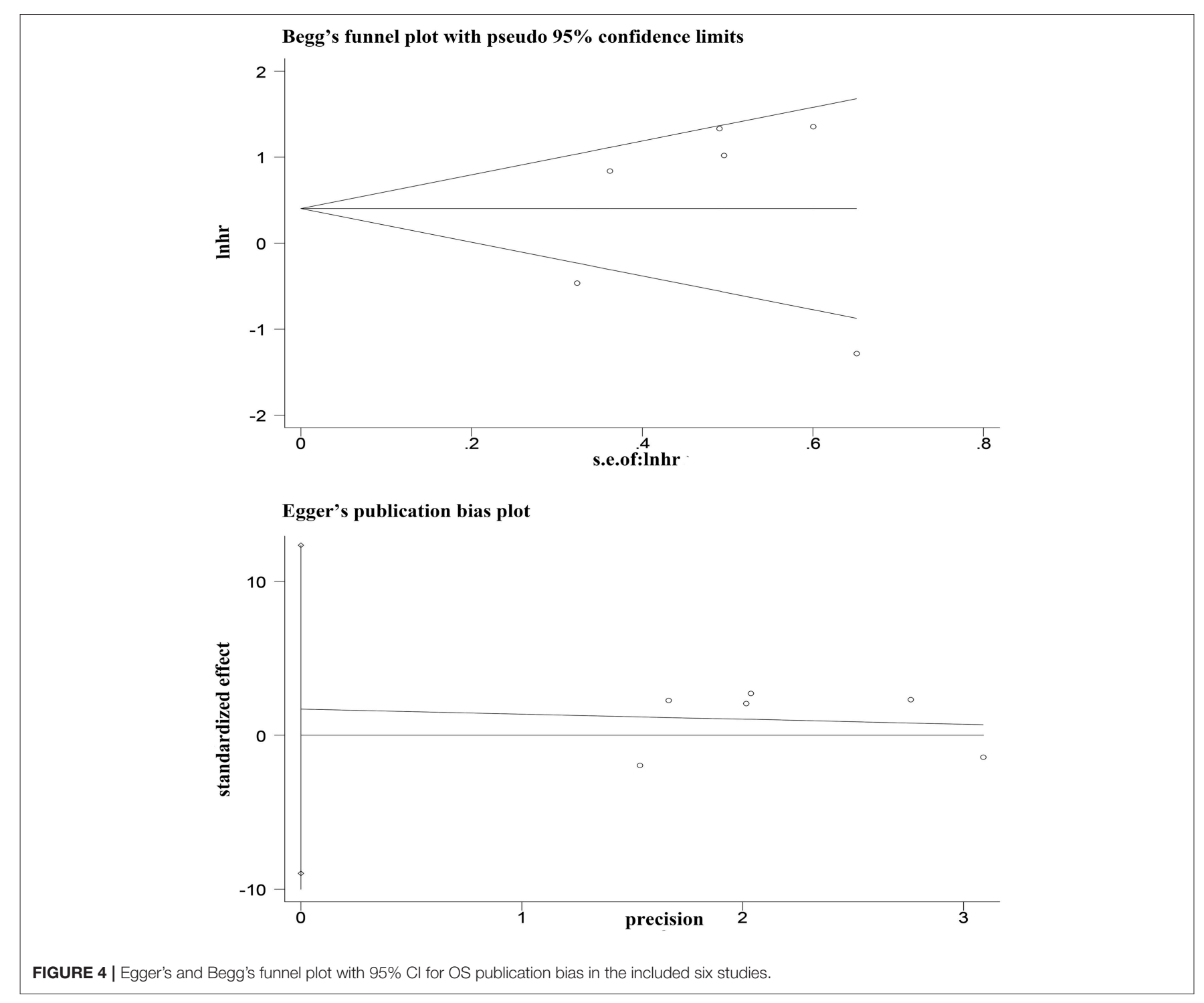

However, MSI tumors in CRC display high infiltration with CD8+ cytotoxic T lymphocytes (CTLs) and activated Th1 cells, which may contribute to better survival (Gubin et al., 2014). MSI tumors are also counterbalanced by upregulating expression of multiple immune checkpoints (Angelova et al., 2015; Becht et al., 2016), such as CTL-associated antigen 4 (CTLA4), PD-1, PD-L1 and indoleamine 2,3-dioxygenase 1 (IDO1). Upregulated after $\mathrm{T}$ cell activation, $\mathrm{PD}-1$ declines when an antigen is cleared. While PD-1 expression remains elevated, as in CRC cancer, $\mathrm{T}$ cells enter a state of exhaustion or anergy (Xiao and Freeman, 2015). A study found that Fusobacterium species could evade the high load of neoantigens in MSI colorectal cancer (Tahara et al., 2014). And these species may facilitate upregulation of PD-L1 and lead to poor survival (Kostic et al., 2013). Considering the dynamic changes of PD-L1 expression, our results showing that PD-L1 expression was significantly associated with poor prognoses appear more credible.
We also noticed a recently literature make a contradictory conclusion with our study. This study considered that no significant differences founded in colorectal cancer-specific or overall survival by Tumor Immunity in the MicroEnvironment (TIME) subtypes (Hamada et al., 2018). We found that the primary data of their study were too old, as one cohort was from 1986 to 1992 and the other was from 1986 to 2004 (Giovannucci et al., 1995; Wark et al., 2009). While, our primary data were carried out from 2006 to 2016. The discrepancies between Hamada et al. (2018) and our study might reflect the different storage time of tissue sections. Reports by Bertheau et al. (1998) and Jacobs et al. (1996), who investigated the loss of immunoreactivity for a panel of antibodies in breast carcinomas, neuroendocrine tumors and lymphomas, indicated that for the majority of epitopes tested there is a time-dependent substantial loss in stored tissue slides. CRC develops via sequential genetic and epigenetic alterations of TCs, and is influenced by tumorhost interactions. Because CRC patients easily developed local 


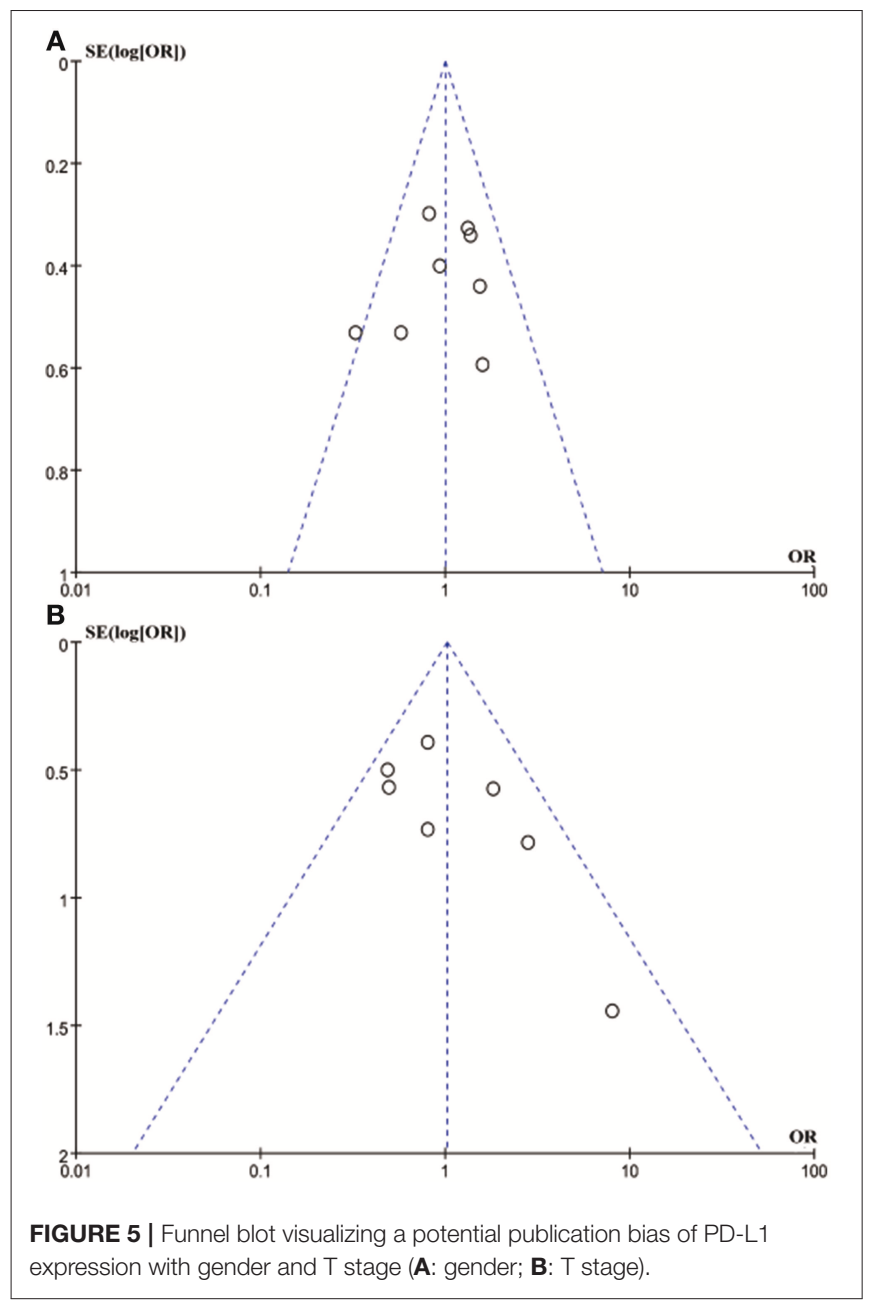

recurrences and distant metastases within 5 years after surgical treatment and CRC has typical immune subgroups (Dienstmann et al., 2017), researchers found that immunotherapy is able to reach center stage in the field of second-line therapy in oncology treatment (Topalian et al., 2012; Hon et al., 2018). As one of the types of CRC, high microsatellite instability (MSI-H) can gather TILs and upregulate PD-L1 expression in tumor cells (Herbst et al., 2014).Currently, PD-L1 expression on TCs is considered as an immune-tolerance mechanism of carcinoma, because it can attract PD-1 expressing immune-inhibitory TILs. However, little is known about the complex interrelationship among PDL1 expression, TILs, and major tumor molecular features. PDL1 promoter methylation (mPD-L1) was significantly correlated with poor PD-L1 mRNA expression, indicating that PD-L1 expression might be regulated by $\mathrm{mPD}-\mathrm{L} 1$ on a cellular level in CRC (Goltz et al., 2016). However, this study was not available to provide data on PD-L1 protein expression and there was a study had published a proteomic characterization of the cohort, showing that protein abundance could not be reliably predicted from DNA- or RNA-level measurements (Zhang et al., 2014). Previous studies have shown a significant correlation of PD-L1 expression with OS in melanoma (Robert et al., 2015), breast cancer (Zhang et al., 2017), renal cell carcinoma
(Motzer et al., 2014), and non-small cell lung cancer (Zhang et al., 2015), and observed prominent clinical benefits of PD-1/PDL1 checkpoint blockades in these carcinoma patients. Although previous trials have suggested no role for immunotherapy in patients with CRC, recent studies have demonstrated that MSI$\mathrm{H}$ in CRC did benefit (Kwak et al., 2016; Overman et al., 2017). Therefore, we investigated the relationship between the expression of PD-L1 and clinicopathological factors, and the results showed that poor differentiation and right colon location in CRC were PD-L1 expression positive. In addition, poor differentiation and right colon location in CRC were also significantly correlated with poor prognoses, which were more likely to be MSI-H. Thus, our study provided a scientific rationale and direct support for clinicians to select MSI-H CRC patients for anti-PD-1/PD-L1 immunotherapy.

This study provided moderate evidence to evaluate the association of PD-L1 expression with prognostic outcomes and clinicopathological factors. However, there were some limitations. Firstly, only six included studies evaluated the association of PD-L1 expression with OS. Although the sample sizes of RFS and DFS were relatively small, their results should have alleviated some of these concerns. Secondly, the cut-off values determining positive and negative PDL1 expression and antibodies for PD-L1 varied among the included studies. Thus, the subgroup of IRS $\geq 4$ had reduced heterogeneity and addressed some of these concerns. Thirdly, only articles published in English were included. Accordingly, to address these limitations, a large multicenter study with uniform evaluation methods (the same antibody and cut-off for positive PD-L1 expression) may be helpful to attain results that are more accurate. Despite the above limitations, the present meta-analysis demonstrated the association of PD-L1 expression with prognostic outcomes and clinicopathological factors. The findings of this study may lead to improvements in the outcomes of anti-PD$1 / \mathrm{PD}-\mathrm{L} 1$ therapy through stratifying patients in a more appropriate manner.

\section{CONCLUSION}

In conclusion, our results showed that $\mathrm{PD}-\mathrm{L} 1$ positive expression might be a new biomarker for poor prognosis in CRC. This information may be helpful for clinicians to stratify CRC patients for anti-PD-1/PD-L1 therapy, especially patients with MSI-H. Well-designed and high-quality studies with uniform evaluation methods are needed to confirm the association of PD-L1 expression in CRC.

\section{AUTHOR CONTRIBUTIONS}

$\mathrm{YL}, \mathrm{MH}$, and LX designed this study. YZ and MH screened identified studies and extracted data. Disagreements were resolved by discussion with YL and YZ performed the statistical analyses. $\mathrm{MH}$ and $\mathrm{YZ}$ prepared the figures and tables. YL, MH, and LX reviewed the results, interpreted the data, and wrote the manuscript. All authors have read and approved the final version of this manuscript. 


\section{REFERENCES}

Angelova, M., Charoentong, P., Hackl, H., Fischer, M. L., Snajder, R., Krogsdam, A. M., et al. (2015). Characterization of the immunophenotypes and antigenomes of colorectal cancers reveals distinct tumor escape mechanisms and novel targets for immunotherapy. Genome Biol. 16:64. doi: 10.1186/s13059-015-0620-6

Becht, E., de Reyniès, A., Giraldo, N. A., Pilati, C., Buttard, B., Lacroix, L., et al. (2016). Immune and stromal classification of colorectal cancer is associated with molecular subtypes and relevant for precision immunotherapy. Clin. Cancer Res. 22, 4057-4066. doi: 10.1158/1078-0432.CCR-15-2879

Bertheau, P., Cazalshatem, D., Meignin, V., de Roquancourt, A., Vérola, O., Lesourd, A., et al. (1998). Variability of immunohistochemical reactivity on stored paraffin slides. J. Clin. Pathol. 51, 370-374. doi: 10.1136/jcp.51.5.370

Dienstmann, R., Vermeulen, L., Guinney, J., Kopetz, S., Tejpar, S., Tabernero, J., et al. (2017). Consensus molecular subtypes and the evolution of precision medicine in colorectal cancer. Nat. Rev. Cancer 17:79. doi: $10.1038 / \mathrm{nrc} .2016 .126$

Droeser, R. A., Hirt, C., Viehl, C. T., Frey, D. M., Nebiker, C., Huber, X., et al. (2013). Clinical impact of programmed cell death ligand 1 expression in colorectal cancer. Eur. J. Cancer 49:233. doi: 10.1016/j.ejca.2013.02.015

Enkhbat, T., Nishi, M., Takasu, C., Yoshikawa, K., Jun, H., Tokunaga, T., et al. (2018). Programmed Cell death ligand 1 expression is an independent prognostic factor in colorectal cancer. Anticancer Res. 38, 3367-3373. doi: 10.21873 / anticanres.12603

Giovannucci, E., Ascherio, A., Rimm, E. B., Colditz, G. A., Stampfer, M. J., and Willett, W. C. (1995). Physical activity, obesity, and risk for colon cancer and adenoma in men. Ann. Intern. Med. 122:327. doi: 10.7326/0003-4819-122-5-199503010-00002

Goltz, D., Gevensleben, H., Dietrich, J., and Dietrich, D. (2016). PD-L1 (CD274) promoter methylation predicts survival in colorectal cancer patients. Onco Immunol. 6:e1257454. doi: 10.1080/2162402X.2016.1257454

Gubin, M. M., Zhang, X., Schuster, H., Caron, E., Ward, J. P., Noguchi, T., et al. (2014). Checkpoint blockade cancer immunotherapy targets tumor-specific mutant antigens. Nature 515, 577-581. doi: 10.1038/nature13988

Hamada, T., Soong, T. R., Masugi, Y., Kosumi, K., Nowak, J. A., da Silva, A., et al. (2018). TIME (Tumor Immunity in the MicroEnvironment) classification based on tumor CD274 (PD-L1) expression status and tumorinfiltrating lymphocytes in colorectal carcinomas. Onco Immunol.7:e1442999. doi: 10.1080/2162402X.2018.1442999

Hansen, J. D., Du Pasquier, L., Lefranc, M. P., Lopez, V., Benmansour, A., and Boudinot, P. (2009). The B7 family of immunoregulatory receptors: a comparative and evolutionary perspective. Mol. Immunol. 46, 457-472. doi: 10.1016/j.molimm.2008.10.007

Herbst, R. S., Soria, J. C., Kowanetz, M., Fine, G. D., Hamid, O., Gordon, M. S., et al. (2014). Predictive correlates of response to the anti-PD-L1 antibody MPDL3280A in cancer patients. Nature 515:563. doi: 10.1038/nature14011

Hon, K. W., Abu, N., Ab Mutalib, N. S., and Jamal, R. (2018). miRNAs and lncRNAs as predictive biomarkers of response to FOLFOX therapy in colorectal cancer. Front. Pharmacol. 9:846. doi: 10.3389/fphar.2018.00846

Inaguma, S., Lasota, J., Wang, Z., Felisiak-Golabek, A., Ikeda, H., and Miettinen, M. (2016). Clinicopathologic profile, immunophenotype, and genotype of CD274 (PD-L1)-positive colorectal carcinomas. Mod. Pathol. 30:278. doi: 10.1038/modpathol.2016.185

Jacobs, T. W., Prioleau, J. E., Stillman, I. E., and Schnitt, S. J. (1996). Loss of tumor marker-immunostaining intensity on stored paraffin slides of breast cancer. J. Natl. Cancer Inst. 88, 1054-1059.doi: 10.1093/jnci/88.15.1054

Keir, M. E., Butte, M. J., Freeman, G. J., and Sharpe, A. H. (2008). PD-1 and its ligands in tolerance and immunity. Ann. Rev. Immunol. 26, 677-704. doi: 10.1146/annurev.immunol.26.021607. 090331

Keir, M. E., Liang, S. C., Guleria, I., Latchman, Y. E., Qipo, A., Albacker, L. A., et al. (2006). Tissue expression of PD-L1 mediates peripheral T cell tolerance. Exp. Med. 2203, 883-895. doi: 10.1084/jem.20051776

Kostic, A. D., Chun, E., Robertson, L., Glickman, J. N., Gallini, C. A., Michaud, M., et al. (2013). Fusobacterium nucleatum potentiates intestinal tumorigenesis and modulates the tumor-immune microenvironment. Cell Host Microbe 14, 207-215. doi: 10.1016/j. chom. 2013. 07.007
Kwak, Y., Koh, J., Kim, D. W., Kang, S. B., Kim, W. H., and Lee, H. S. (2016) Immunoscore encompassing $\mathrm{CD} 3+$ and $\mathrm{CD} 8+\mathrm{T}$ cell densities in distant metastasis is a robust prognostic marker for advanced colorectal cancer. Oncotarget 7, 81778-81790. doi: 10.18632/oncotarget. 13207

Lee, K. S., Kim, B. H., Oh, H. K., Kim, D. W., Kang, S. B., Kim, H., et al. (2018). Programmed cell death ligand-1 protein expression and CD274/PD-L1 gene amplification in colorectal cancer: implications for prognosis. Cancer Sci. 109, 2957-2969. doi: 10.1111/cas.13716

Lee, L. H., Cavalcanti, M. S., Segal, N. H., Hechtman, J. F., Weiser, M. R., Smith, J. J., et al. (2016). Patterns and prognostic relevance of PD1 and PD-L1 expression in colorectal carcinoma. Modern Pathol. 29:1433. doi: 10.1038/modpathol.2016.139

Lee, S. J., Jun, S. Y., Lee, I. H., Kang, B. W., Park, S. Y., Kim, H. J., et al. (2018) CD274, LAG3, and IDO1 expressions in tumor-infiltrating immune cells as prognostic biomarker for patients with MSI-high colon cancer. J. Cancer Res. Clin. Oncol. 144, 1005-1014. doi: 10.1007/s00432-018-2620-x

Li, Y., Lei, L., Dai, W., Cai, G., Xu, Y., Li, X., et al. (2016). Prognostic impact of programed cell death-1 (PD-1) and PD-ligand 1 (PD-L1) expression in cancer cells and tumor infiltrating lymphocytes in colorectal cancer. Mol. Cancer 15:55. doi: 10.1186/s12943-016-0539-X

Liu, R., Peng, K., Yu, Y., Liang, L., Xu, X., Li, W., et al. (2018). Prognostic value of immunoscore and PD-L1 expression in metastatic colorectal cancer patients with different RAS status after palliative operation. BioMed Res. Int. 2018, 1-8. doi: 10.1155/2018/5920608

Lu, B., Chen, L., Liu, L., Zhu, Y., Wu, C., Jiang, J., et al. (2011). T-cell-mediated tumor immune surveillance and expression of B7 co-inhibitory molecules in cancers of the upper gastrointestinal tract. Immunol. Res. 50, 269-275. doi: 10.1007/s12026 - 011-8227-9

Lussier, D. M., Johnson, J. L., Hingorani, P., and Blattman, J. N. (2015). Combination immunotherapy with $\alpha$-CTLA- 4 and $\alpha$-PD-L1 antibody blockade prevents immune escape and leads to complete control of metastatic osteosarcoma. J. Immunother. Cancer 3:21. doi: 10.1186/s40425-015-0067-Z

Motzer, R. J., Rini, B. I., McDermott, D. F., Redman, B. G., Kuzel, T. M., Harrison, M. R., et al. (2014). Nivolumab for metastatic renal cell carcinoma: results of a randomized phase II trial. J. Clin. Oncol. 33, 1430-1437. doi: $10.1200 /$ JCO.2014.59.0703

Okazaki, T., and Honjo, T. (2006). The PD-1-PD-L pathway in immunological tolerance. Trends Immunol. 27, 195-201. doi: 10.1016/j.it.2006.02.001

Overman, M. J., McDermott, R., Leach, J. L., Lonardi, S., Lenz, H. J., Morse, M. A., et al. (2017). Nivolumab in patients with metastatic DNA mismatch repair-deficient or microsatellite instability-high colorectal cancer (CheckMate 142): an open-label, multicentre, phase 2 study. Lancet Oncol. 18:1182. doi: 10.1016/S1470-2045(17)30422-9

Robert, C., Long, G. V., Brady, B., Dutriaux, C., Maio, M., Mortier, L., et al. (2015). Nivolumab in previously untreated melanoma without BRAF mutation. $N$. Engl. J. Med. 372, 320-330. doi: 10.1056/NEJMoa1412082

Schalper, K. A., Velcheti, V., Carvajal, D., Wimberly, H., Brown, J., Pusztai, L., et al. (2014). In situ tumor PD-L1 mRNA expression is associated with increased TILs and better outcome in breast carcinomas. Clin. Cancer Res. 20, 2773-2782 doi: 10.1158/1078-0432.CCR-13-2702

Shi, S. J., Wang, L. J., Wang, G. D., Guo, Z. Y., Wei, M., Meng, Y. L., et al. (2013). B7-H1 Expression is associated with poor prognosis in colorectal carcinoma and regulates the proliferation and invasion of HCT116 colorectal cancer cells. PLoS ONE 8:e76012. doi: 10.1371/journal.pone.0076012

Siegel, R. L., Miller, K. D., Fedewa, S. A., Ahnen, D. J., Meester, R. G. S., Barzi, A., et al. (2017). Colorectal cancer statistics. CA Cancer J. Clin. 67, 104-117. doi: $10.3322 /$ caac. 21395

Stang, A. (2010). Critical evaluation of the Newcastle-Ottawa scale for the assessment of the quality of nonrandomized studies in meta analyses. Eur. J. Epidemiol. 25, 603-605. doi: 10.1007/s10654-010-9491-z

Tahara, T., Yamamoto, E., Suzuki, H., Maruyama, R., Chung, W., Garriga, J., et al. (2014). Fusobacterium in colonic flora and molecular features of colorectal carcinoma. Cancer Res. 74, 1311-1318. doi: 10.1158/0008-5472.CAN13-1865

Toh, J. W. T., de Souza, P., Lim, S. H., Singh, P., Chua, W., Ng, W., et al. (2016). The potential value of immunotherapy in colorectal cancers: review of the evidence for programmed death-1 inhibitor therapy. Clin. Colorec. Cancer 15:285. doi: $10.1016 /$ j.clcc. 2016.07 .007 
Topalian, S. L., Hodi, F. S., Brahmer, J. R., Gettinger, S. N., Smith, D. C., McDermott, D. F., et al. (2012). Safety, activity, and immune correlates of anti-PD-1 antibody in cancer. N. Engl. J. Med. 366, 2443-2454. doi: 10.1056/NEJMoa1200690

Wang, A., Wang, H. Y., Liu, Y., Zhao, M. C., Zhang, H. J., Lu, Z. Y., et al. (2015). The prognostic value of PD-L1 expression for non-small cell lung cancer patients: a meta-analysis. Eur. J. Surg. Oncol. 41, 450-456. doi: 10.1016/j.ejso.2015.01.020

Wang, L., Liu, Z., Fisher, K. W., Ren, F., Lv, J., Davidson, D. D., et al. (2017). Prognostic value of programmed death ligand 1, p53, and Ki-67 in patients with advanced stage colorectal cancer. Hum. Pathol.71, 20-29. doi: 10.1016/j.humpath.2017.07.014

Wang, L., Ren, F., Wang, Q., Baldridge, L. A., Monn, M. F., Fisher, K. W., et al. (2016). Significance of programmed death ligand 1 (PD-L1) immunohistochemical expression in colorectal cancer. Mol. Diagn. Ther. 20, 175-181. doi: 10.1007/s40291-0160188-1

Wang, Y., Wang, H., Yao, H., Li, C., Fang, J. Y., and Xu, J. (2018). Regulation of PDL1: emerging routes for targeting tumor immune evasion. Front. Pharmacol. 19:536. doi: 10.3389/fphar.2018.00536

Wark, P. A., Wu, K., van 't Veer, P., Fuchs, C. F., and Giovannucci, E. L. (2009). Family history of colorectal cancer: a determinant of advanced adenoma stage or adenoma multiplicity? Int. J. Cancer 125, 413-420. doi: 10.1002/ijc.24288

Welch, H. G., and Robertson, D. J. (2016). Colorectal cancer on the decline - why screening can't explain it all. N. Engl. J. Med. 374:1605. doi: 10.1056/NEJMp1600448

Xiao, Y., and Freeman, G. J. (2015). The microsatellite instable subset of colorectal cancer is a particularly good candidate for checkpoint blockade immunotherapy. Cancer Discov. 5:16. doi: 10.1158/2159-8290.CD14- 1397
Zhang, B., Wang, J., Wang, X., Zhu, J., Liu, Q., Shi, Z., et al. (2014). Proteogenomic characterization of human colon and rectal cancer. Nature 513, 382-387. doi: 10.1038/nature13438

Zhang, M., Sun, H., Zhao, S., Wang, Y., Pu, H., Wang, Y., et al. (2017). Expression of PD-L1 and prognosis in breast cancer: a meta-analysis. Oncotarget 8 , 31347-31354. doi: 10.18632/oncotarget.15532

Zhang, Y., Kang, S., Shen, J., He, J., Jiang, L., Wang, W., et al. (2015). Prognostic significance of progracmmed cell death 1 (PD-1) or PD-1 ligand 1 (PD-L1) expression in epithelial-originated cancer: a meta-analysis. Medicine 94:e515. doi: 10.1097/MD.0000000000000515

Zheng, P., and Zhou, Z. (2015). Human cancer immunotherapy with PD-1/PD-L1 blockade. Biomark. Cancer 7, 15-18. doi: 10.4137/BIC.S29325

Zhu, H., Qin, H., Huang, Z., Li, S., Zhu, X., He, J., et al. (2015). Clinical significance of programmed death ligand-1 (PD-L1) in colorectal serrated adenocarcinoma. Int. J. Clin. Exp. Pathol. 8, 9351-9359.

Zou, W., Wolchok, J. D., and Chen, L. (2016). PD-L1 (B7-H1) and PD-1 pathway blockade for cancer therapy: mechanisms, response biomarkers and combinations. Sci. Transl. Med. 8:328rv4. doi: 10.1126/scitranslmed.aad7118

Conflict of Interest Statement: The authors declare that the research was conducted in the absence of any commercial or financial relationships that could be construed as a potential conflict of interest.

Copyright (C) 2019 Li, He, Zhou, Yang, Wei, Bian, Christopher and Xie. This is an open-access article distributed under the terms of the Creative Commons Attribution License (CC BY). The use, distribution or reproduction in other forums is permitted, provided the original author(s) and the copyright owner(s) are credited and that the original publication in this journal is cited, in accordance with accepted academic practice. No use, distribution or reproduction is permitted which does not comply with these terms. 